\title{
The Hot White Dwarf Population in the EUVE Survey
}

\author{
STÉPHANE VENNES \\ Center for EUV Astrophysics, 2150 Kittredge Street, \\ University of California at Berkeley, Berkeley, CA 94720-5030, USA
}

The processes leading to the formation of white dwarf stars are known only in their most general principles; post-asymptotic giant branch evolution, leading to the formation of $\mathrm{C}-\mathrm{O}$ degenerate cores, is possibly the main formation channel of white dwarf stars. In contrast, observations of hot white dwarf stars and studies of their main population characteristics offer detailed insights into the origin and evolution of these objects. We examine some new facts uncovered in the study of the survey of hot white dwarf stars at extreme ultraviolet (EUV) wavelengths. We describe model atmosphere techniques required to interpret these observations and discuss some implications of our findings for stellar evolution theory.

\section{The White Dwarf Population}

The white dwarf population has been surveyed using various means and methodologies, often using proper motion surveys (e.g., Luyten catalogs) or color sensitive surveys (e.g., Palomar-Green \& Montréal-Cambridge-Tololo catalogs). Bergeron, Saffer, \& Liebert (1992; BSL) completed an analysis of the sample of hydrogen-rich white dwarfs compiled by McCook \& Sion (1987) and established some of their properties; they noted in particular the extreme narrowness of the mass distribution. On the other hand, the temperature distribution, or alternatively the luminosity function, of hot white dwarf stars $\left(T_{\text {eff }} \geq 25,000 \mathrm{~K}\right.$ ) is not well established (see Fleming, Liebert, \& Green 1986). The atmospheric composition of hot white dwarf stars has been the subject of a number of investigations and theoretical studies. Apart from the existence of two parallel channels, helium- and hydrogen-rich (see MacDonald \& Vennes 1991), it has also been established that heavy elements are found in larger concentrations in the hottest white dwarfs (see Vennes \& Fontaine 1992). Although diffusion in white dwarf atmospheric layers is extremely efficient, agreement between theory and observation has not yet been achieved (see Chayer et al. 1995).

The Extreme Ultraviolet Explorer (EUVE) survey of hot white dwarf stars is the most comprehensive collection of such objects (Bowyer et al. 1994). A variety of phenomena are represented in the sample and such occurrences are useful in characterizing the white dwarf population, its origin and destiny. I will describe in the following sections a comprehensive research program aimed at a complete description of the white dwarf stars detected at EUV wavelengths. To achieve this goal, we have obtained optical spectroscopy for most objects in the sample; these data are primarily used to describe the mass distribution and space density of the white dwarfs in the survey for comparison with evolutionary scenarios ( $\$ 2$ ). I also describe far ultraviolet (FUV) and EUV spectroscopy of representative objects. These data help determine the chemical structure of the atmospheric layers of hot white dwarf stars (\$3). Additional EUV spectroscopic data are also presented and analyzed by Dupuis \& Vennes (1995a,b). Many white dwarfs in the EUV sample are found in binaries, some in short-period pairs, and we briefly examine the properties of these systems and possible implications for evolutionary scenarios $(\$ 4)$. We summarize our findings in $\S 5$. 

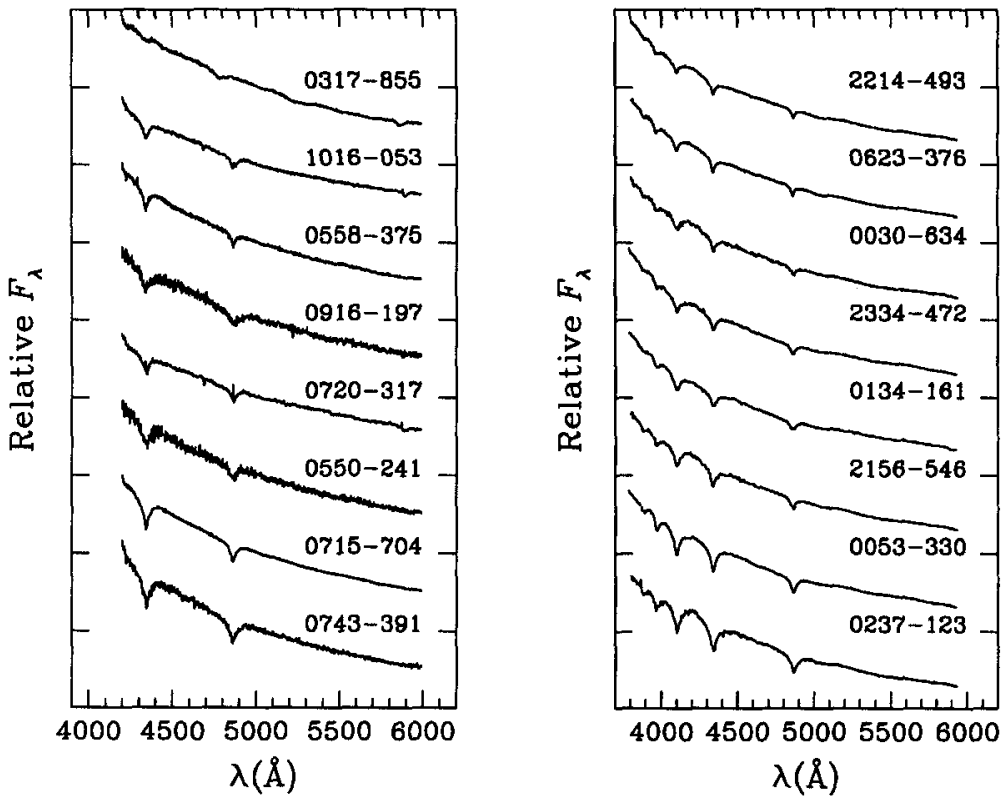

FIGURE 1. Optical spectra of southern-hemisphere white dwarfs obtained at CTIO (Vennes et al. 1995d). The white dwarfs are ordered, from top to bottom, with decreasing temperatures. Note the composite DAO+dM spectra of EUVE J0720-317 and EUVE J1016-053. The star EUVE J0317-855 is a new magnetic white dwarf (DAp).

\section{Surface Gravity and Temperature}

Over a hundred white dwarf stars have been detected in the EUVE all-sky survey. We have obtained follow-up optical spectroscopy for a sample subset at the MichiganMIT-Dartmouth (MDM) observatory, the Lick observatory, and the Cerro-Tololo InterAmerican (CTIO) observatory. I present in Figure 1 some remarkable spectra obtained at CTIO. The measured effective temperatures range approximately from $60 \times 10^{3} \mathrm{~K}$ (top) down to $30-40 \times 10^{3} \mathrm{~K}$ (bottom). In particular, both EUVE J0743-391 and EUVE J0916-197 have high surface gravities and masses in excess of $1 M_{\odot}$. We also noted the DAO+dM composite spectra of EUVE J0720-317 and EUVE J1016-053; further investigations revealed short orbital periods (Vennes \& Thorstensen 1994; Thorstensen, Vennes, \& Bowyer 1996). These objects are commonly viewed as recent survivors of common-envelope evolution (see de Kool \& Ritter 1993). The peculiar spectrum of EUVE J0317-855 shows evidence of a large magnetic field. Other magnetic white dwarfs have been detected in the EUVE survey, most notably PG 1658+441 (=EUVE J1659+440), and population statistics can be derived from the complete sample.

We have determined the effective temperature and surface gravity for 57 white dwarfs from the EUVE sample by modeling Balmer line profiles (Vennes et al. 1995d). We used techniques similar to BSL. I present in Figure 2 (top panel) the surface gravity distribution of our sample and compare it to BSL's sample. Two main differences are apparent: the BSL distribution is significantly sharper than the $E U V E$ distribution and a larger number of high-gravity stars are identified in the EUVE sample. The former is 

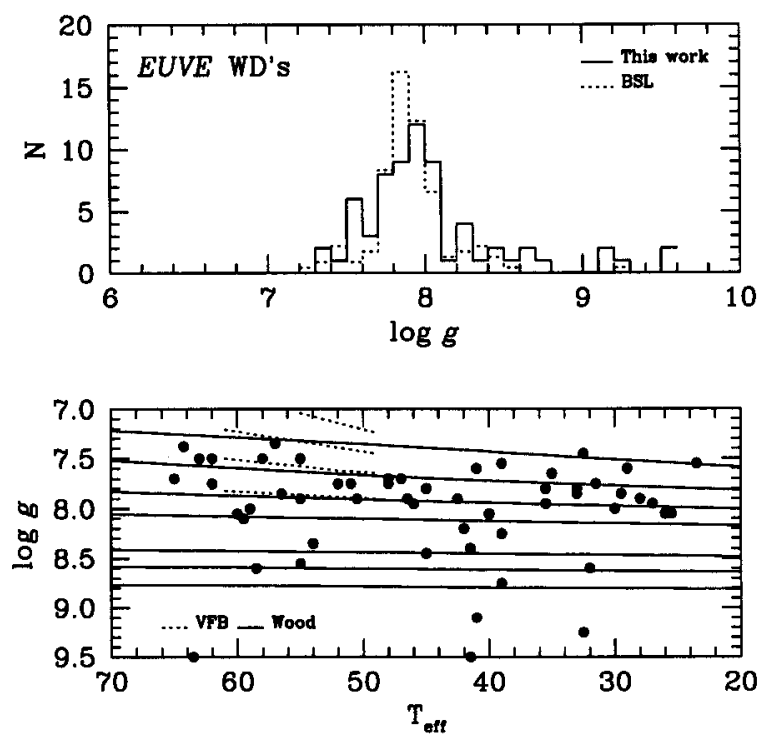

FIGURE 2. (top panel) Surface gravity distribution of a sample of $\sim 60$ hot DA white dwarfs discovered in the EUVE survey. The wide temperature range explored with EUVE explains the broader distribution relative to the "cooler" sample studied by Bergeron, Saffer, \& Liebert (1992; BSL). (lower panel) Surface gravity as a function of effective temperature compared to theoretical mass radius relations at $0.4,0.45,0.5$ and $0.6 M_{\odot}$ (VFB; Vennes, Fontaine, \& Brassard 1995), and 0.4, 0.5, 0.6, 0.7, 0.9,1.0 and $1.1 M_{\odot}$ (Wood; Wood 1995). Note the low-mass $\left(M \leq 0.4 M_{\odot}\right)$ and high-mass $\left(M \geq 1.0 M_{\odot}\right)$ white dwarfs identified in the survey.

easily explained by the relative homogeneity of the BSL sample which is composed of cooler objects, while the $E U V E$ sample extends up to $\sim 65 \times 10^{3} \mathrm{~K}$ where a significant radius increase is predicted. The second difference is more fundamental and is one of the main surprises in the $E U V E$ survey: several new high-mass white dwarfs have been discovered, four of them exceeding $1.2 M_{\odot}$. Such a large turn-out may offer support to a merger model, in which degenerate cores in a close binary merge into a single massive white dwarf. The measured temperatures and surface gravities are compared to theoretical mass-radius relations in the lower panel. The mass distribution, using Wood's mass-radius relations, is presented in Figure 3 and compared with BSL's distribution. The $E U V E$ distribution is significantly broader which is attributed to a lesser accuracy in gravity determinations of extremely hot white dwarfs. To summarize, we find that the average masses of the $E U V E$ and BSL samples are identical $\left(<M>\sim 0.55 M_{\odot}\right)$. We find that eight objects in the $E U V E$ sample exceed $1.0 M_{\odot}$ while two of them are below $0.4 M_{\odot}$ and are possibly the results of binary evolution.

\section{Chemical Composition}

The extreme diversity of white dwarf chemical compositions is already evident in the classification nomenclature: DA (by number, $X \sim 1$ and $\left.Y, Z \leq 10^{-5}\right)$, DO $(X \leq 0.5$, $Y \geq 0.5$ ), DB $\left(X \leq 10^{-5}, Y \sim 1\right.$ ), etc. (see MacDonald \& Vennes 1991). What became apparent only in the EUVE survey is the extreme diversity within the hydrogen-rich DA class itself. Following the EUVE photometric survey (completed in 1993 January, see 


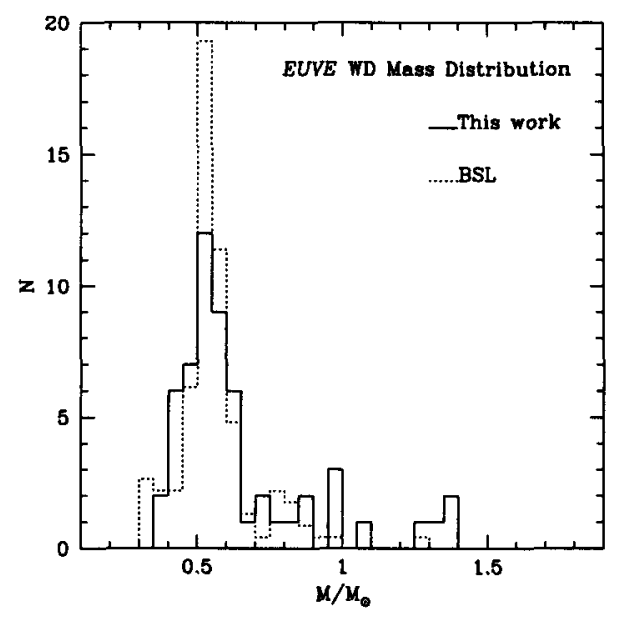

FIGURE 3. Mass distribution of hydrogen-rich white dwarfs in the EUVE survey. The EUVE distribution is compared to a renormalized BSL's distribution; several massive white dwarfs were discovered in the EUVE survey.

Finley 1995), a systematic program of EUV spectroscopic observations demonstrated for the first time the extent of abundance variations in white dwarf atmospheres. I illustrate this phenomenon in Figure 4 (left panel) using two extreme cases with metallicity ranging between upper limits of $Z \leq 10^{-8}(\mathrm{HZ} 43)$ and actual measurements near $Z=0.00002$ (MCT 0455-2812). Both objects are separated by less than $10^{4} \mathrm{~K}$ (i.e., separated in age by less than $10^{6} \mathrm{yrs}$ ) and yet show metallicity variations of a factor of more than a thousand. The distinction between helium- and hydrogen-rich spectra is illustrated by a comparison with the helium-rich star MCT 0501-2858 (Vennes et al. 1994).

The effect of metallicity on the measured energy distribution of hydrogen-rich stars is also predicted using model spectra (right panel). We computed model atmospheres in radiative equilibrium including detailed opacities of $\mathrm{C}, \mathrm{N}, \mathrm{O}, \mathrm{Si}$ and $\mathrm{Fe}$ based on Opacity Project radiative cross-sections (see Pradhan 1995). The abundance ratios amount to a metallicity of $Z=0.00002$ with iron accounting for $50 \%$ of the heavy element concentration. This high-metallicity model displays a steep downturn near $260 \AA$ relative to the pure-hydrogen model. We interpret the helium-rich white dwarf MCT 0455-2858 with a model at $T_{\text {eff }}=70 \times 10^{3} \mathrm{~K}, \log g=7$ and abundances of $\mathrm{C} / \mathrm{He}=0.8 \%, \mathrm{~N} / \mathrm{He}=0.01 \%$, and $\mathrm{O} / \mathrm{He}=0.02 \%$. Our knowledge of the true atmospheric composition of these objects (MCT 0455-2812 and MCT 0501-2858) and similar cases (e.g., G191 B2B) is, at best, fragmentary.

An exhaustive description of the atmospheric composition of a hot white dwarf is not available yet; high-dispersion FUV spectra currently offer the most detailed abundance estimates in hot white dwarf stars. The case of G191 B2B is quite exemplary: using International Ultraviolet Explorer and ORFEUS data (Fig. 5) we have assembled the most complete description of a hot white dwarf atmosphere yet. I summarize in Table 1 the abundances measured in G191 B2B. The emergence of Fe as the most abundant trace element in hot white dwarf atmospheres only reinforces the need for accurate atomic data for this element (e.g., Iron Project, see Pradhan 1995). Considerable discrepancy is observed between diffusion model predictions and abundance measurements. It is now 


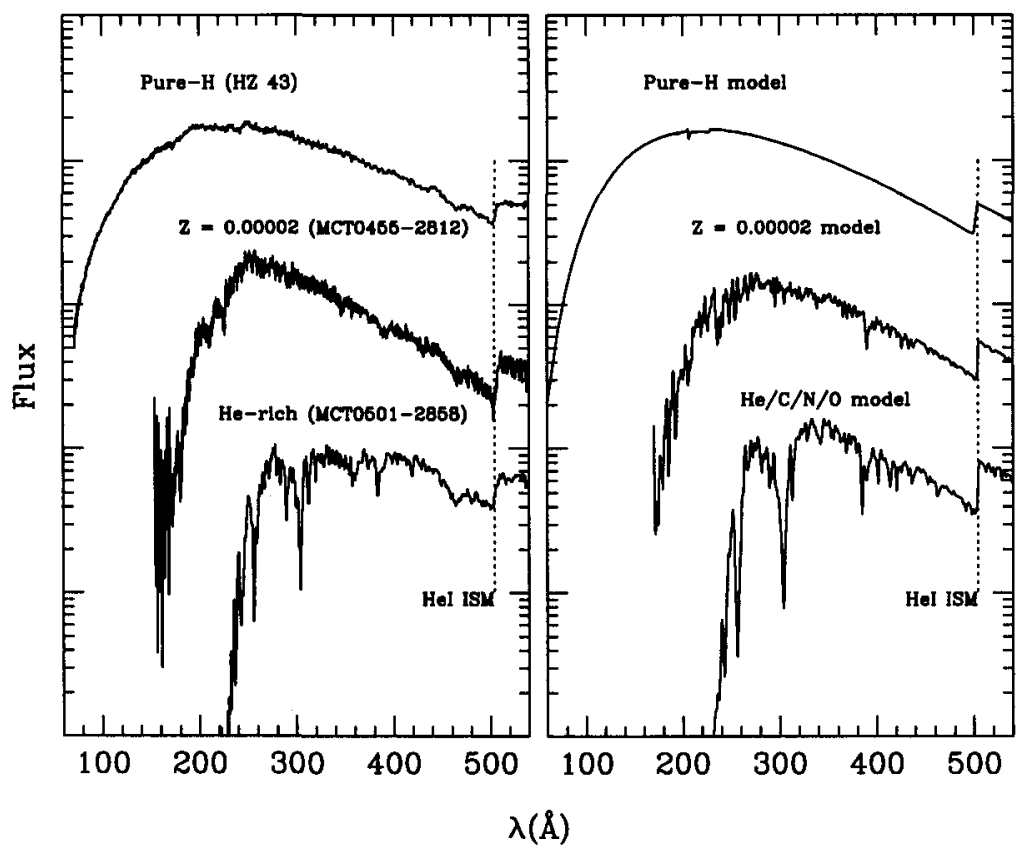

FIGURE 4. A comparison between actual EUVE spectroscopy (left panel) and model spectra using Opacity Project radiative cross-sections (see Pradhan 1995) (right panel). We show three spectra, each representative of a class of white dwarfs: HZ $\mathbf{4 3}$ for pure-hydrogen objects, MCT 0455-2812 for high-metallicity white dwarfs, and MCT 0501-2858 for helium-rich objects.

acknowledged that the observed abundance pattern may be dictated by yet unidentified fundamental mechanisms that operate in these atmospheres. Some suggest the presence of a weak mass loss (see Chayer et al. 1995).

\section{Binaries}

The white dwarf population in the EUV spectral range shows a substantial fraction of white dwarfs in binaries, some with very short orbital periods-evidence for past interaction-and most in wide pairs. Vennes \& Thorstensen $(1994,1995)$ noted the presence in EUV all-sky surveys of a class of short-period binaries that emerge from a common envelope phase (de Kool \& Ritter 1993). Detailed studies of BD+08 102 (K V+DA; Kellett et al. 1995), HR 1608 and HR 8210 (K0 IV+DA and A8+DA, respectively; Landsman, Simon, \& Bergeron 1993; Wonnacott, Kellett, \& Stickland 1993), HD 33959C (F+DA; Hodgkin et al. 1993), and $\beta$ Crt (A+DA; Fleming et al. 1991) all demonstrate the importance of a multiwavelength approach to optical identification of EUV sources. Both $\beta$ Crt and HD 33959C are candidate close binaries. The study of binaries with white dwarf companions may help constrain models of close-binary evolution as well as models of binary star formation; a well-defined sample of these objects may help resolve the issue of mass-correlation in binaries. We also examine interaction mechanisms between close binary components: in particular, the peculiar helium enrichment 
TABLE 1. Photospheric composition of the hot DA white dwarf G191 B2B.

\begin{tabular}{ccl} 
Element & $\mathrm{Z} / \mathrm{H}$ & References \\
$\mathrm{C}$ & $2 \times 10^{-6}$ & Vennes et al. (1991) \\
$\mathrm{N}$ & $3 \times 10^{-6}$ & Vennes et al. (1991) \\
$\mathrm{O}$ & $1 \times 10^{-6}$ & Vennes et al. (1995a) \\
$\mathrm{Si}$ & $3 \times 10^{-7}$ & Vennes et al. (1991) \\
$\mathrm{P}$ & $1 \times 10^{-8}$ & Vennes et al. (1995a) \\
$\mathrm{S}$ & $1 \times 10^{-7}$ & Vennes et al. (1995a) \\
$\mathrm{Fe}$ & $3 \times 10^{-6}$ & Vennes et al. (1992), Holberg et al. (1994), Werner \& Dreizler (1994) \\
$\mathrm{Ni}$ & $1 \times 10^{-6}$ & Holberg et al. (1994), Werner \& Dreizler (1994) \\
$\mathrm{He}$ & $\leq 2 \times 10^{-4}$ & Vennes et al. (1995a) \\
$\mathrm{Cl}$ & $\leq 3 \times 10^{-9}$ & Vennes et al. (1995a) \\
\hline
\end{tabular}

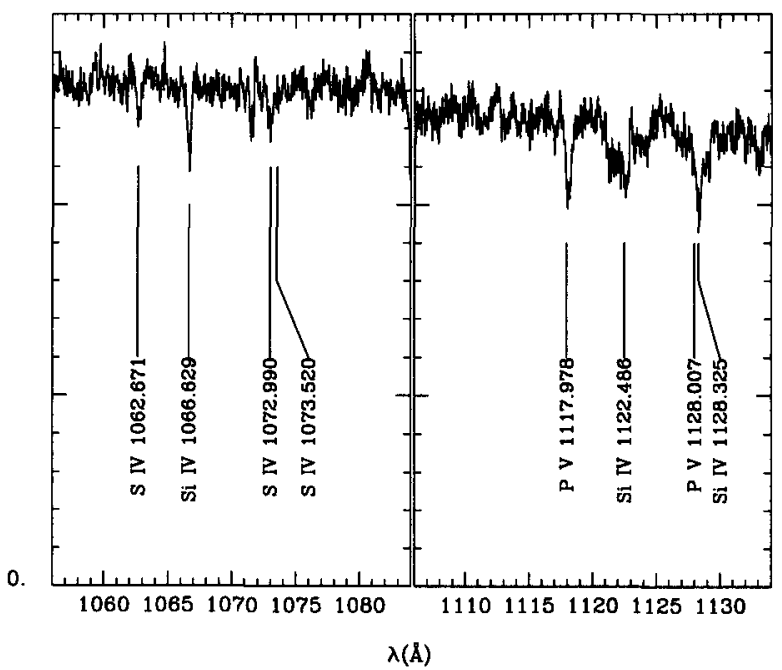

FIGURE 5. Silicon, sulfur and phosphorus in ORFEUS (see Hurwitz \& Bowyer 1995) spectra of G191 B2B. Similar detections have been made in the atmosphere of MCT 0455-2812. The silicon abundance is consistent with previsous measurements obtained with IUE (Vennes, Thejll, \& Shipman 1991). New abundances of $\mathrm{P} / \mathrm{H}=1 \times 10^{-8}$ and $\mathrm{S} / \mathrm{H}=1 \times 10^{-7}$ have been secured with the ORFEUS data (Vennes et al. 1995a).

of the white dwarf is possibly the spectroscopic signature of mass exchange between a mass-losing red dwarf and an accreting white dwarf.

As noted above, the optical spectrum of WD+MS binaries is often dominated by luminous main sequence stars. New ultraviolet (UV) observations of late-type stars detected in the $E U V E$ all-sky survey revealed an unsuspected white dwarf companion to the K0 star HD18131 (Fig. 6). The International Ultraviolet Explorer (IUE) spectrum shows a composite of a white dwarf and a late-type star. The white dwarf dominates the emission below $2000 \AA$ while the K0 star prevails at longer wavelengths. A model atmosphere analysis of the new ultraviolet spectrophotometry and of the EUV photometry reveals a 


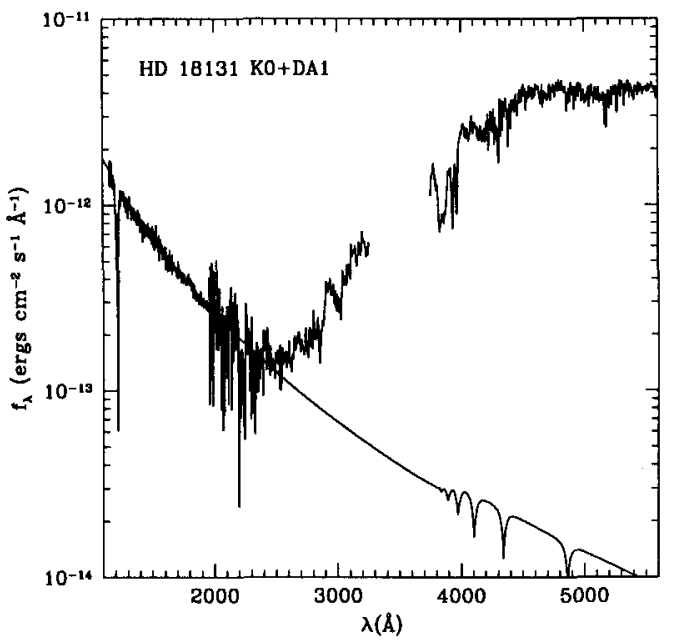

FIGURE 6. Ultraviolet and optical energy distribution of the Ko IV+DA1 binary HD 18131. The hot white dwarf binary is identified with the EUV source EUVE J0254-053 (Vennes et al. $1995 \mathrm{c})$.

hot, hydrogen-rich (DA) white dwarf $\left(T_{\text {eff }} \sim 30,000 \mathrm{~K}\right)$ that is the most likely source of the EUV emission (EUVE J0254-053). Optical spectroscopy revealed that the K0 star is a subgiant (K0 IV) and most likely constitutes with the white dwarf a wide pair at a distance of $\sim 70 \mathrm{pc}$. This discovery has important implications for the EUV white dwarf population survey and, in particular, for the binary frequency.

The binary HD 18131 shares some properties with the binary HR 1608 (Landsman et al. 1993) with the distinction that HR 1608's K0 star has a measured orbital velocity of $5 \mathrm{~km} \mathrm{~s}^{-1}$. Both components of these systems are evolved, i.e., a young white dwarf and a subgiant, and because the components in each system were presumably formed together, they share a similar evolutionary age, and therefore a similar initial mass. The sample of binaries comprised of one or two evolved components offers important insights to the formation and evolution of stars. In particular we find evidence that, in cases like HR 1608 and HD 18131, the mass distribution in binaries tends to favor components with similar mass. This mass correlation between components allows us, in turn, to identify cases that establish initial mass-final mass relations for white dwarf stars (Weidemann \& Koester 1984). The case of HD 18131 requires further investigation, but we note that the adopted atmospheric parameters for the white dwarf are consistent with a mass of $0.4-0.6 M_{\odot}$ and that the progenitor was possibly a $\mathrm{G}$ star with a mass of $\sim 1.3 M_{\odot}$.

The hot white dwarf in the close binary EUVE J1016-053 has been classified a DAO white dwarf; this mixed $\mathrm{H} / \mathrm{He}$ composition has been attributed to steady accretion from its close red dwarf companion (as in EUVE J2013+400; see Thorstensen, Vennes, \& Shambrook 1994). Spectroscopic observations obtained with EUVE also show the effect of heavy element opacities on the white dwarf EUV energy distribution (Fig. 7). Spectral synthesis including trace opacities (see Pradhan 1995) of helium and a group of heavy elements $(\mathrm{C}, \mathrm{N}, \mathrm{O}, \mathrm{Si}, \mathrm{S}, \mathrm{Fe})$ in the otherwise hydrogen-rich atmosphere constrain their abundance to $\mathrm{Y} / \mathrm{Y}_{\odot}=\mathrm{Z} / \mathrm{Z}_{\odot}=2 \times 10^{-3}$, in support of a simple accretion model. The low 


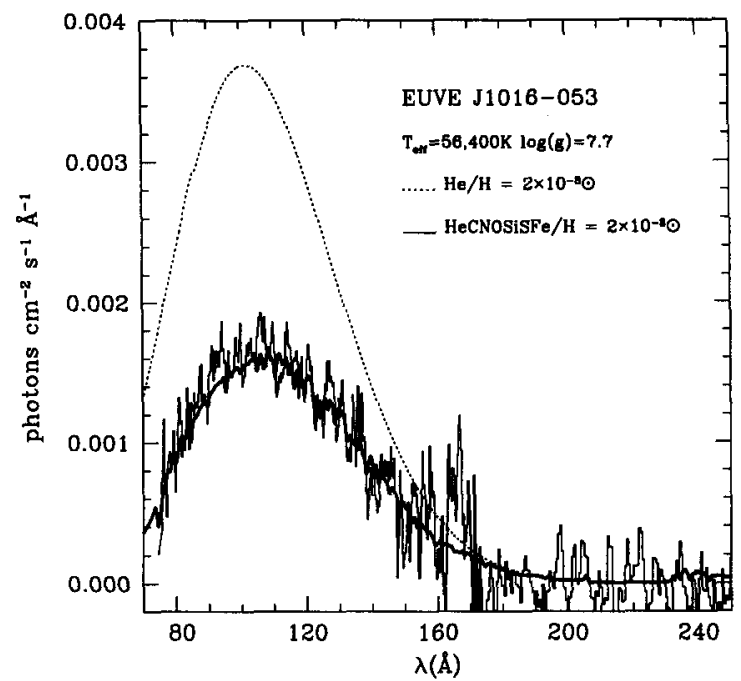

FIGURE 7. EUV spectrum of the white dwarf in the close binary EUVE J1016-053. The respective effects of helium and heavy element opacities are compared in two model simulations. The white dwarf is probably accreting from its companion at a low rate of $10^{-18} M_{\odot} \mathrm{yr}^{-1}$ (Vennes et al. 1995b).

surface-averaged helium abundance measured in the white dwarf atmosphere limits the accretion rate to $10^{-19}-10^{-18} M_{\odot} \mathrm{yr}^{-1}$, i.e., much lower than the Bondi-Hoyle accretion rate which is of the same order as the red dwarf mass loss rate $\left(\geq 10^{-14} M_{\odot} \mathrm{yr}^{-1}\right)$, therefore invalidating a simple wind-accretion model. We speculate that interaction with a weak white dwarf mass loss or a magnetosphere may inhibit accretion onto the white dwarf. Accretion of heavy elements may also be restricted to smaller areas possibly associated with magnetic poles.

EUV spectroscopy of the hot, hydrogen-rich white dwarf GD 50 reveals an unusual photospheric mixture of hydrogen and helium (Fig. 8). This hot DA white dwarf is also remarkable for its mass $\left(\approx 1.3 M_{\odot}\right)$ near the Chandrasekhar limit. The spectra obtained with EUVE show a prominent He II resonance line series and constrain its atmospheric parameters to $T_{\text {eff }}=40,300 \pm 100 \mathrm{~K}$ and $n(\mathrm{He}) / n(\mathrm{H})=2.4 \pm 0.1 \times 10^{-4}$ (assuming $\log g=9.0$ ). The optical photometry excludes the presence of a companion earlier than dM7-8. The presence of helium in an isolated, massive DA white dwarf is paradoxical; radiative levitation of helium is clearly undone by the high surface gravity; we exclude the possibility that helium is accreted from the ISM at the Bondi-Hoyle rate. Because accretion of ISM material onto GD 50 would constitute a unique occurrence and because we find no traces of heavier elements, we examine an alternative. If massive white dwarfs do result from binary evolution with a stellar merger, large orbital angular momentum may be transferred to rotation angular momentum which may induce large meridional circulation current, possibly dredging-up helium from the envelope.

\section{Conclusions}

The EUVE survey of the white dwarf population and the follow-up multi-wavelength spectroscopic observations, revealed unsuspected classes of objects and phenomena. Fol- 


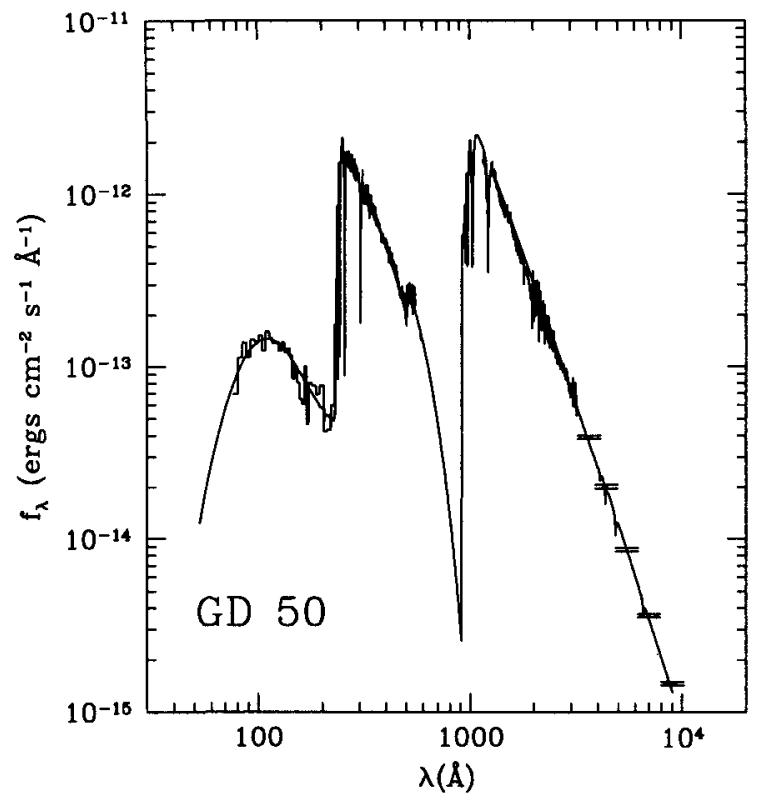

FIGURE 8. Complete energy distribution (EUVE, IUE, and Landolt's UBVRI photometry) of the massive DA white dwarf GD 50. Note the depression below $911 \AA$ associated with neutral hydrogen absorption in the local ISM. The dramatic effect of the helium concentration $\left(\mathrm{He} / \mathrm{H}=2.4 \times 10^{-4}\right)$ is evidenced by strong HeII ground state transitions. We also constrain the local interstellar medium column densities of neutral helium and hydrogen to $n_{\mathrm{He}} \mathrm{I}=6 \times 10^{16}$ and $n_{\mathrm{H}} \mathrm{I}=9 \times 10^{17} \mathrm{~cm}^{-2}$. (Vennes, Dupuis, \& Bowyer 1995).

lowing optical spectroscopic observations we have discovered new massive $\left(M \geq 1.0 M_{\odot}\right)$ white dwarfs, some of which, like GD 50, may be the outcome of binary evolution and stellar mergers. The class of EUV-emitting white dwarfs displays large abundance variations with a metallicity index ranging from $Z \leq 10^{-8}$ up to $Z=0.00002$. Dupuis \& Vennes (1995a,b) analyzed EUV spectroscopic observations of a large sample of hot DA white dwarfs and presented evidence of an abrupt decline in the metallicity index near a temperature of $50,000 \mathrm{~K}$. This effect may be the spectroscopic signature of a weak mass loss. The EUVE sample of white dwarfs also displays the effects of binary evolution with the discovery of several new binaries and some post-common-envelope systems. Vennes \& Thorstensen $(1994,1995)$ summarize the properties of these systems. We found evidence of interaction between components of close binaries: the EUV spectrum of the white dwarf in EUVE J1016-053 displays traces of heavy elements, possibly accreted from the red dwarf companion. This work is supported by NASA contract NAS5-30180 and NASA grant NAG5-2405.

\section{REFERENCES}

Bergeron, P., SAfFer, R., \& Liebert, J. 1992, ApJ, 394, 228 BSL

Bowyer, S., Lieu, R., Lampton, M., Lewis, J., Wu, X., Drake, J. J., \& Malina, R. F. 
1994, ApJS, 93, 569

Chayer, P., Vennes, S., Pradhan, A. K., Thejll, P., Beauchamp, A., Fontaine, G., \& WESEMAEL, F. 1995, these proceedings

DE, Kool, M., \& RitTer, H. 1993, A\&A, 267, 397

Dupurs, J., \& VenNes, S. 1995a, in Lecture Notes in Physics 443, ed. D. Koester \& K. Werner Berlin: Springer, 323

DuPUis, J., \& VeNNES, S. 1995b, these proceedings

FinLeY, D. 1995, these proceedings

Fleming, T. A., Lebert, J., \& Green, R. F. 1986, ApJ, 308, 176

Fleming, T. A., Schmitt, J. H. M. M., Barstow, M. A., \& Mittaz, J. P. D. 1991, A\&A, 246, L47

Hodgkin, S. T., Barstow, M. A., Fleming, T. A., Monier, R., \& Pye, J. P. 1993, MNRAS, 263, 229

Holberg, J. B., Hubeny, I., Barstow, M. A., Lanz, T., Sion, E. M., \& Tweedy, R. W. 1994, ApJ, 425, L105

HURwitz, M., \& BowYER, S. 1995, these proceedings

KelletT, B. J., ET AL. 1995, ApJ, 438, 364

LANDOLT, A. U. 1992, AJ, 104, 340

Landsman, W., Simon, T., \& Bergeron, P. 1993, PASP, 105, 841

MacDonald, J., \& VenNes, S. 1991, ApJ, 371, 719

McCooK, G. P., \& Sion, E. M. 1987, ApJS, 65, 603

PradHAN, A. K. 1995, these proceedings

Thorstensen, J. R., Vennes, S., \& Bowyer, S. 1996, ApJ, 457, in press

Thorstensen, J. R., Vennes, S., \& Shambrook, A. 1994, AJ, 108, 1924

Vennes, S., Chayer, P., Thorstensen, J. R., Bowyer, S., \& Shipman, H. L. 1992, ApJ, 392, L27

Vennes, S., Chayer, P., Hurwitz, M., \& Bowyer, S. 1995a, ApJ, submitted

Vennes, S., Dupuis, J., \& BowYer, S. 1995, ApJ, submitted

Vennes, S., Dupuis, J., Bowyer, S., Fontaine, G., Wiercigroch, A., Jelinsky, P., WeSEMAEL, F., \& MaLina, R. F. 1994, ApJ, 421, L35

Vennes, S., Dupuis, J., Bowyer, S., \& Pradhan, A. K. 1995b, ApJ, submitted

Vennes, S., \& Fontaine, G. 1992, ApJ, 401, 288

Vennes, S., Fontaine, G., \& Brassard, P. 1995, A\&A, 296, 117

Vennes, S., Mathioudakis, M., Doyle, J. G., Thorstensen, J. R., \& Byrne, P. B. 1995c, A\&A, 299, L29

Vennes, S., Thejll, P., Dupuis, J., Génova, R., Thorstensen, J. R., Fontaine, G., Wesemael, F., \& LAMONTAGNE, R. 1995d, ApJ, in preparation

Vennes, S., ThEJll, P., \& ShIPMAN, H. L. 1991, in White Dwarfs, ed. G. Vauclair \& E.M. Sion, Dordrecht: Kluwer, 235

Vennes, S., \& Thorstensen, J. R. 1994, ApJ, 433, L29

Vennes, S., \& Thorstensen, J. R. 1995, in Lecture Notes in Physics 443, ed. D. Koester \& K. Werner, Berlin: Springer, 313

WememanN, V., Koester, D. 1984, A\&A, 132, 195

Werner, K., \& Dreizler, S. 1994, A\&A, 286, L31

WonnacotT, D., Kellett, B. J., \& Stickland, D. J. 1993, MNRAS, 262, 277

Wood, M. A. 1995, in Lecture Notes in Physics 443, ed. D. Koester \& K. Werner, Berlin: Springer, in press 